M. Itô and M. Nishio

Nagoya Math. J.

Vol. 115 (1989), 1-22

\title{
POINCARÉ TYPE CONDITIONS OF THE REGULARITY FOR THE PARABOLIC OPERATOR OF ORDER $\alpha$
}

\author{
MASAYUKI ITÔ AND MASAHARU NISHIO
}

\section{$\S 1$. Introduction}

Let $R^{n+1}=R^{n} \times R$ denote the $(n+1)$-dimensional Euclidean space $(n \geqq 1)$. For $X \in R^{n+1}$, we write $X=(x, t)$ with $x \in R^{n}$ and $t \in R$. In this case, $R^{n}$ is called the $x$-space of $R^{n+1}=R^{n} \times R$.

For an $\alpha$ with $0<\alpha<1$, we write

$$
L^{(\alpha)}=\frac{\partial}{\partial t}+(-\Delta)^{\alpha}
$$

where $(-\Delta)^{\alpha}$ is the fractional power of the Laplacian $-\Delta=-\sum_{j=1}^{n} \frac{\partial^{2}}{\partial x_{j}^{2}}$ on the $x$-space. In the case of $\alpha=1 / 2, L^{(1 / 2)}$ is called the Poisson operator on $R^{n+1}$.

First we shall examine some properties of the elementary solution $W^{(\alpha)}$ of $L^{(\alpha)}$. By using the reduced functions with respect to $W^{(\alpha)}$, we shall show the existence of swept-out measures with respect to $W^{(a)}$. By using swept-out measures, we shall give the notion of the regularity for boundary points of an open set in $R^{n+1}$.

The purpose of this paper is to give a Poincaré type condition for the regularity of boundary points of an open set in $R^{n+1}$.

Our main theorem is the following

TheOREM. Let $\Omega$ be an open set in $R^{n+1}$ and $X$ a boundary point of $\Omega$. If there exists a non-empty open set $\omega$ in the $x$-space whose $\alpha$-tusk $T_{X}^{(\alpha)}(\omega)$ at $X$ is in $C \Omega$, then $X$ is regular for the Dirichlet problem of $L^{(\alpha)}$ on $\Omega$.

For an $X=(x, t) \in R^{n+1}$ and an open set $\omega$ in the $x$-space, the $\alpha$-tusk $T_{X}^{(\alpha)}(\omega)$ of $\omega$ at $X$ is defined by

$$
T_{X}^{(\alpha)}(\omega)=\left\{\left(x+p y, t-p^{2 \alpha}\right) ; y \in \omega, 0<p<p_{0}\right\}
$$

Received January 14, 1988. 
with some $p_{0}>0$.

For the heat equation, E. G. Effros and J. L. Kazdan [3] discussed a similar Poincaré type condition of the regularity.

\section{§2. Superparabolic functions and the Riesz decomposition}

Let $C_{K}^{\infty}\left(R^{k}\right)$ denote the usual topological vector space of all infinitely differentiable functions on $R^{k}$ with compact support $(k \geqq 1)$. For $0<\alpha<1$, we recall the fractional power $(-\Delta)^{\alpha}$ of $-\Delta$ on the $x$-space $R^{n} ;(-\Delta)^{\alpha}$ is the convolution operator on $R^{n}$ defined by the distribution $-C_{n, \alpha}$ p.f. $|x|^{-n-2 \alpha}$, where $|x|$ denotes the distance between $x$ and the origin 0 in $R^{n}$ and $C_{n, \alpha}=-4^{\alpha} \pi^{-n / 2} \Gamma((n+2 \alpha) / 2) / \Gamma(-\alpha)$, that is,

$$
\text { p.f. }|x|^{-n-2 \alpha}(\phi)=\lim _{\delta \downarrow 0} \int_{|x|>\delta}(\phi(x)-\phi(0))|x|^{-n-2 \alpha} d x
$$

for every $\phi \in C_{K}^{\infty}\left(R^{n}\right)$.

We denote by $\left(g_{t}\right)_{t \geqq 0}$ the Gaussian semi-group on $R^{n}$, namely $g_{t}(x)=$ $(4 \pi t)^{-n / 2} \exp \left(-|x|^{2} / 4 t\right)(t>0)$, and $g_{0}=\varepsilon$. Here we denote by $\varepsilon$ the Dirac measure at the origin of $R^{k}$ for every $k \geqq 1$. Put

$$
W^{(\alpha)}(X)= \begin{cases}(2 \pi)^{-n} \int_{R^{n}} \exp \left(-t|\xi|^{2 \alpha}+i x \cdot \xi\right) d \xi & t>0 \\ 0 & t \leqq 0,\end{cases}
$$

where $X=(x, t)$ and $x \cdot \xi$ denotes the inner product on $R^{n}$. By means of the Fourier transform, we see easily that $W^{(\alpha)}$ (resp. $\left.\tilde{W}^{(\alpha)}\right)$ is the elementary solution of $L^{(\alpha)}$ (resp. $\tilde{L}^{(\alpha)}$ ), where $\tilde{W}^{(\alpha)}(x, t)=W^{(\alpha)}(x,-t)$ and $\tilde{L}^{(\alpha)}=-\partial / \partial t$ $+(-\Delta)^{\alpha}$ (see for example [4]). Let $\left(\sigma_{t}^{\alpha}\right)_{t \geqq 0}$ be the one-sided stable semigroup of order $\alpha$ on $R^{+}$, where $R^{+}$denotes the semi-group of all nonnegative numbers. Then for any $t>0$ and $x \in R^{n}$,

$$
W^{(\alpha)}(x, t)=\int_{0}^{\infty} g_{s}(x) d \sigma_{t}^{\alpha}(s)>0
$$

(see [1], p. 74), $\int_{R^{n}} W^{(\alpha)}(x, t) d x=1$ and $W^{(\alpha)}(x, t)$ is a decreasing function of $|x|$. Put

$$
\psi_{\alpha}(t)=W^{(\alpha)}((1,0, \cdots, 0), t)
$$

then we have easily

$$
W^{(\alpha)}(x, t)=|x|^{-n} \psi_{\alpha}\left(t|x|^{-i \sigma}\right)
$$

LEMMA 2.1. $\psi_{\alpha}(t)=O(t)$ as $t \downarrow 0$. 
Proof. Let $\nu$ be the uniform measure on the unit sphere $\left\{x \in R^{n}\right.$; $|x|=1\}$ with $\int d \nu=1$. Denoting by $\hat{\nu}$ the Fourier transform of $\nu$, we have

$$
\psi_{\alpha}(t)=(2 \pi)^{-n / 2} \int_{R^{n}} \exp \left(-t|\xi|^{2 \alpha}\right) \hat{\nu}(\xi) d \xi, \quad \lim _{t \downarrow 0} \psi_{\alpha}(t)=0
$$

and

$$
\begin{aligned}
\frac{d}{d t} \psi_{\alpha}(t) & =(2 \pi)^{-n / 2} \int_{R^{n}}\left(-|\xi|^{2 \alpha} \exp \left(-t|\xi|^{2 \alpha}\right) \hat{\nu}(\xi)\right) d \xi \\
& =(2 \pi)^{-n / 2} \int_{0}^{\infty} \int_{R^{n}}\left(-|\xi|^{2 \alpha} \exp \left(-s|\xi|^{2}\right) \hat{\nu}(\xi)\right) d \xi d \sigma_{t}^{\alpha}(s)
\end{aligned}
$$

for $t>0$ (see (2.1)). Let $\phi \in C_{K}^{\infty}\left(R^{n}\right)$ satisfying $0 \leqq \phi \leqq 1$, supp $[\phi] \subset\left\{x \in R^{n}\right.$; $|x|<1\}$ and $\phi=1$ on a neighborhood of 0 , where supp $[\phi]$ denotes the support of $\phi$. For any $s>0$, we have

$$
\begin{aligned}
\int_{R^{n}}|\xi|^{2 \alpha} & \exp \left(-s|\xi|^{2}\right) \hat{\nu}(\xi) d \xi=(2 \pi)^{n / 2}(-\Delta)^{\alpha}\left(g_{s} * \nu\right)(0) \\
= & (2 \pi)^{n / 2} C_{n, \alpha-1}\left(|x|^{-n-2 \alpha+2}\right) *\left(\Delta g_{s}\right) * \nu(0) \\
= & (2 \pi)^{n / 2} C_{n, \alpha-1}\left(\phi(x)|x|^{-n-2 \alpha+2}\right) *\left(\Delta g_{s}\right) * \nu(0) \\
& +(2 \pi)^{n / 2} C_{n, \alpha-1}\left(\Delta\left((1-\phi(x))|x|^{-n-2 \alpha+2}\right)\right) *\left(g_{s}\right) * \nu(0) .
\end{aligned}
$$

Since $0 \notin \operatorname{supp}\left[\left(\phi(x)|x|^{-n-2 \alpha}\right) * \nu\right]$ and $\Delta g_{s}$ vanishes uniformly outside any neighborhood of 0 ,

$$
\lim _{s \downarrow 0}\left(\phi(x)|x|^{-n-2 \alpha+2}\right) *\left(\Delta g_{s}\right) * \nu(0)=0 .
$$

Therefore the function $\int_{R^{n}}|\xi|^{2 \alpha} \exp \left(-s|\xi|^{2}\right) \hat{\nu}(\xi) d \xi$ of $s$ is bounded on $(0, \infty)$, so that $(d / d t) \psi_{\alpha}(t)$ is bounded on $(0, \infty)$, which shows Lemma 2.1 .

Let $\left(P_{t}^{(\alpha)}\right)_{t \geqq 0}$ be the convolution semi-group whose infinitesimal generator is equal to $-L^{(\alpha)}\left(\right.$ see $\left.[7]^{1)}\right)$; then

$$
P_{s}^{(\alpha)} * u(x, t)=\int_{R^{n}} W^{(\alpha)}(x-y, s) u(y, t-s) d y
$$

for every $u \in C_{K}\left(R^{n+1}\right)$, where $C_{K}\left(R^{n+1}\right)$ denotes the usual topological vector space of all finite continuous functions on $R^{n+1}$ with compact support. For a non-negative continuous function $\phi(t)$ on $(0, \infty)$, we put

$$
W_{(\phi)}^{(\alpha)}(x, t)==\phi(t) W^{(\alpha)}(x, t) .
$$

1) Evidently $-L^{(\alpha)}$ is a generalized Laplacian, that is, for any $\phi \in C_{K}^{\infty}\left(R^{n+1}\right)$ with $\phi \geqq 0$ and $\phi(0)=\max _{X \in R^{n+1}} \phi(X),-\left(L^{(\alpha)} \phi\right)(0) \leqq 0$. 
For a sequence $\left(\phi_{m}\right)_{m=1}^{\infty}$ in $C_{K}((0, \infty))$ with $\phi_{m} \geqq 0, \int \phi_{m} d t=1$ and with $\operatorname{supp}\left[\phi_{m}\right] \subset\left((m+1)^{-1}, m^{-1}\right)$, we shall often use the sequence $\left(W_{\left(\phi_{m}\right)}^{(\alpha)}\right)_{m=1}^{\infty}$. We say that such a $\left(\phi_{m}\right)_{m=1}^{\infty}$ is an approximate sequence of the Dirac measure.

Definition 1. A non-negative function $u$ on $R^{n+1}$ is said to be superparabolic of order $\alpha$ if the following two conditions are satisfied:

(1) $u$ is lower semi-continuous on $R^{n+1}$ and $u<\infty$ a.e..

(2) For any $s \geqq 0, u \geqq P_{s}^{(\alpha)} * u$ on $R^{n+1}$.

We denote by $S_{\alpha}$ (resp. $S_{\alpha, c}$ ) the set of all superparabolic (resp. all continuous superparabolic) functions of order $\alpha$, and by $\tilde{S}_{\alpha}$ (resp. $\tilde{S}_{\alpha, c}$ ) the set of all functions $u$ with $\tilde{u} \in S_{\alpha}$ (resp. $\tilde{u} \in S_{\alpha, c}$ ), where $\tilde{u}(x, t)=u(x,-t)$.

For a non-negative Borel measure $\mu$ on $R^{n+1}$, we denote by $W^{(\alpha)} \mu$ (resp. $\tilde{W}^{(\alpha)} \mu$ ) the function defined by the convolution $W^{(\alpha)} * \mu$ (resp. $\tilde{W}^{(\alpha)} * \mu$ ) and call it the $W^{(\alpha)}$-potential (resp. the $\tilde{W}^{(\alpha)}$-potential) of $\mu$.

Remark 2.2. (1) $1 \in S_{\alpha, c}$ and for $u \in S_{\alpha}, u$ is locally integrable.

(2) The condition (2) in Definition 1 is equivalent to $u \geqq W_{(\phi)}^{(\alpha)} * u$ for every $\phi \in C_{K}((0, \infty))$ with $\phi \geqq 0$ and $\int \phi d t=1$.

(3) If $W^{(\alpha)} \mu<\infty$ (resp. $\tilde{W}^{(\alpha)} \mu<\infty$ ) a.e., then $W^{(\alpha)} \mu \in S_{\alpha}$ (resp. $\left.\tilde{W}^{(\alpha)} \mu \in \tilde{S}_{\alpha}\right)$.

We denote by $M_{\alpha}$ (resp. $M_{\alpha, c}, \tilde{M}_{\alpha}$ and $\tilde{M}_{\alpha, c}$ ) the set of all positive Borel measures $\mu$ with $W^{(\alpha)} \mu \in S_{\alpha}$ (resp. $W^{(\alpha)} \mu \in S_{\alpha, c}, \tilde{W}^{(\alpha)} \mu \in \tilde{S}_{\alpha}$ and $\tilde{W}^{(\alpha)} \mu \in \tilde{S}_{\alpha, c}$ ). For a Borel measure $\mu$ and a Borel set $A$, we denote by $\left.\mu\right|_{A}$ the Borel measure defined by $\left.\mu\right|_{A}(E)=\mu(A \cap E)$ for every Borel set $E$.

Lemma 2.3. For $u \in S_{\alpha}$, we have

$$
\int_{a}^{b} \int_{|x| \geqq 1} u(x, t)|x|^{-n-2 \alpha} d x d t<\infty
$$

for every finite interval $[a, b]$.

Proof. Let $\phi \in C_{K}^{\infty}\left(R^{n+1}\right)$ with $0 \leqq \phi \leqq 1, \phi(X)=1$ on $\{X=(x, t) ;|x| \leqq 1 / 2$, $a \leqq t \leqq b\}$ and with $\phi(X)=0$ on $\{X=(x, t) ;|x| \geqq 3 / 4\}$. Since for any $X=(x, t) \in C \operatorname{supp}[\phi]$,

$$
\tilde{L}^{(\alpha)} \phi(x, t)=-C_{n, \alpha} \int_{R^{n}} \phi(y, t)|x-y|^{-n-2 \alpha} d y \leqq 0,
$$

$\operatorname{supp}\left[\left(\tilde{L}^{(\alpha)} \phi\right)^{+}\right] \subset \operatorname{supp}[\phi]$. On the other hand for any open ball $B$ con- 
taining supp $[\phi]$,

$$
\begin{aligned}
\int_{B} u\left(\tilde{L}^{(\alpha)} \phi\right) d X & =\lim _{s \downarrow 0} \int_{B} u \frac{\phi-\tilde{P}_{s}^{(\alpha)} * \phi}{s} d X \\
& =\lim _{s \downarrow 0}\left(\int_{R^{n+1}} \frac{u-P_{s}^{(\alpha)} * u}{s} \phi d X+\int_{C B} u \frac{\tilde{P}_{s}^{(\alpha)} * \phi}{s} d X\right) \geqq 0,
\end{aligned}
$$

where $\tilde{P}_{s}^{(\alpha)}$ is defined by $\int f d \tilde{P}_{s}^{(\alpha)}=\int f(-X) d P_{s}^{(\alpha)}(X)$ for every $f \in C_{K}\left(R^{n+1}\right)$. Hence

$$
\begin{aligned}
\infty & >\int_{R^{n+1}} u\left(\tilde{L^{(\alpha)}} \phi\right)^{+} d X \geqq \int_{R^{n+1}} u\left(L^{(\alpha)} \phi\right)^{-} d X \\
& \geqq \int_{a}^{b} \int_{|x| \geqq 1} u(x, t)\left(C_{n, \alpha} \int_{R^{n}} \phi(y, t)|x-y|^{-n-2 \alpha} d y\right) d x d t \\
& \geqq 2^{-n-2 \alpha} C_{n, \alpha} \int_{|y| \leqq 1 / 2} d y \int_{a}^{b} \int_{|x| \geqq 1} u(x, t)|x|^{-n-\_\alpha} d x d t
\end{aligned}
$$

which shows Lemma 2.3.

Lemma 2.4. (1) $S_{\alpha}$ and $S_{\alpha, c}$ are convex semi-lattices by $u \wedge v(X)=$ $\min (u(X), v(X))$.

(2) Let $u \in S_{\alpha}$ and let $\left(\phi_{m}\right)_{m=1}^{\infty}$ be an approximate sequence of the Dirac measure. Then $W_{\left(\phi_{m}\right)}^{(\alpha)} * u \in S_{\alpha, c}$ and $W_{\left(\phi_{m}\right)}^{(\alpha)} * u \uparrow u$ with $m \uparrow \infty$.

(3) Let $u, v \in S_{\alpha}$ and $\omega$ be an open set in $R^{n+1}$. If $u \leqq v$ a.e. on $\omega$, then $u \leqq v$ on $\omega$.

Proof. The assertion (1) is evident (see Definition 1). Since $W_{\left(\phi_{m}\right)}^{(\alpha)}$ is finite continuous, Lemmas 2.1, 2.3 give $W_{\left(\phi_{m}\right)}^{(\alpha)} * u \in S_{\alpha, c}$. Since $\left(W_{\left(\phi_{m}\right)}^{(\alpha)}(X) d X\right)_{m=1}^{\infty}$ converges vaguely to $\varepsilon$ as $m \rightarrow \infty$, we have the second part of (2) (see Definition 1). The assertion (3) follows from (2).

LEMMA 2.5. For $u \in S_{\alpha}$, the family $\left(\frac{u-P_{s}^{(\alpha)} * u}{s} d X\right)_{s>0}$ of positive measures converges vaguely as $s \downarrow 0$, where $d X$ denotes the Lebesgue measure on $R^{n+1}$. Denote by $\mu$ its vague limit. Then

$$
\int_{R^{n+1}} u \tilde{L^{(\alpha)}} \phi d X=\int_{R^{n+1}} \phi d \mu
$$

for every $\phi \in C_{K}^{\infty}\left(R^{n+1}\right)$.

Proof. For any $\phi \in C_{K}^{\infty}\left(R^{n+1}\right)$ with $\phi \geqq 0$, we take $r>0$ with supp [ $\left.\phi\right]$ $\subset\{X ;|X| \leqq r\}$. For $(x, t) \in R^{n+1}$ with $|x| \geqq 2 r$ and any $s>0$, Lemma 2.1 shows 


$$
\begin{aligned}
\mid \underline{\phi(x, t)-\tilde{P}_{s}^{(a)} * \phi(x, t)} & \leqq \frac{1}{s} \int W^{(\alpha)}(x-y, s) \phi(y, t+s) d y \\
& \leqq \frac{1}{s} \int W^{(\alpha)}\left(\frac{x}{2}, s\right) \phi(y, t+s) d y \\
& \leqq C|x|^{-n-2 \alpha}
\end{aligned}
$$

for some constant $C$. The Lebesgue theorem and Lemma 2.3 give

$$
\int u \tilde{L}^{(\alpha)} \phi d X=\lim _{s \downarrow 0} \int \frac{u-P_{s}^{(\alpha)} * u}{s} \phi d X
$$

Hence $\left(\frac{i u-P_{s}^{(\alpha)} * u}{s} d X\right)_{s>0}$ converges vaguely as $s \downarrow 0$ and we get

$$
\int u \tilde{L}^{(\alpha)} \phi d X=\int \phi d \mu
$$

for every $\phi \in C_{K}^{\infty}\left(R^{n+1}\right)$.

The above positive Borel measure $\mu$ is called the associated measure of $u$.

Remark 2.6. Let $\mu \in M_{\alpha}$. Then the associated measure of $W^{(\alpha)} \mu$ is equal to $\mu$, because $\frac{W^{(\alpha)} \mu-P_{s}^{(\alpha)} * W^{(\alpha)} \mu}{s}=\frac{1}{s} \int_{0}^{s} P_{t}^{(\alpha)} * \mu d t^{2)}$ (see (2.2)).

LEMMA 2.7. Let $u \in S_{\alpha},\left(u_{m}\right)_{m=1}^{\infty} a$ sequence in $S_{\alpha}, \mu$ the associated measure of $u$ and $\mu_{m}$ the associated measure of $u_{m}(m \geqq 1)$. If $\lim _{m \rightarrow \infty} u_{m}=u$ a.e. and if there exixts $v \in S_{\alpha}$ such that for any $m \geqq 1, u_{m} \leqq v$, then $(\mu)_{m=1}^{\infty}$ converges vaguely to $\mu$ as $m \rightarrow \infty$.

Proof. For any $\phi \in C_{K}^{\infty}\left(R^{n+1}\right)$ with $\phi \geqq 0$, Lemmas 2.3, 2.5 and $\int v\left|\tilde{L}^{(\alpha)} \phi\right| d X<\infty$ give

$$
\int \phi d \mu=\int u \tilde{L}^{(\alpha)} \phi d X=\lim _{m \rightarrow \infty} u_{m} \tilde{L}^{(\alpha)} \phi d X=\lim _{m \rightarrow \infty} \int \phi d \mu_{m},
$$

which shows Lemma 2.7.

LEMMA 2.8. Let $u$ be a non-negative continuous function on $R^{n+1}$. If $u=P_{s}^{(\alpha)} * u$ for every $s>0, u$ is constant.

For the proof, we use the following

Lemma 2.9 (Choquet-Deny [2]). Let $\sigma$ be a positive Borel measure on

2) $\int_{0}^{s} P_{t}^{(\alpha)} * \mu d t$ is a positive measure defined by $\int_{0}^{s} \phi d\left(P_{t}^{(\alpha)} * \mu\right) d t$ for every $\phi \in C_{K}\left(R^{n+1}\right)$. 
$R^{k}(k \geqq 1)$ with $\int d \sigma=1$ and $h$ a non-negative Borel function on $R^{k}$. Assume that $R^{k}$ is generated by $\operatorname{supp}[\sigma]$ as a group and that $h * \sigma=h$ on $R^{k}$. Then $h$ has the following representation:

$$
h(x)=\int \exp (a \cdot x) d \nu(a) \text { a.e. }
$$

with some positive Borel measure $\nu$ on $R^{k}$.

Proof of Lemma 2.8. Let $\phi$ be a non-negative continuous function on $(0, \infty)$ with compact support and with $\int \phi(t) d t=1$. Then $u=P_{s}^{(\alpha)} * u$ give $u=W_{(\phi)}^{(\alpha)} * u$. Applying Lemma 2.9 with $\sigma=W_{(\phi)}^{(\alpha)}$, we see that there exists a positive measure $\nu$ on $R^{n+1}$ such that

$$
u(x, t)=\int_{R^{n+1}} \exp (a \cdot x+b t) d \nu(a, b) \text { a.e. }
$$

By Lemma 2.3, we have

$$
\int_{R^{n+1}} \int_{|x| \geq 1} \exp (a \cdot x+b t)|x|^{-n-2 \alpha} d x d \nu(a, b)<\infty,
$$

so that $\operatorname{supp}[\nu] \subset\{0\} \times R$. By using $u=P_{s}^{(\alpha)} * u$ for every $s>0$ again, we conclude that $u$ is constant.

Proposition 2.10. Let $u \in S_{\alpha}$ and the associated measure of $u$. Then

$$
u=W^{(\alpha)} \mu+c \quad \text { on } R^{n+1}
$$

with some constant $c \geqq 0$. Furthermore if for any positive Borel measure $\nu$ on $R^{n+1}, u-W^{(\alpha)} \nu=a$ a.e. with some constant $a$, then $\nu=\mu$ and $a=c$.

Proof. For a positive integer $m$, we put $\mu_{m}=\left.\mu\right|_{B(0, m)}$, where $B(C, m)$ denotes the open ball in $R^{n+1}$ with center 0 and with radius $m$. For $\phi \in C_{K}^{\infty}\left(R^{n+1}\right)$ with $\phi \geqq 0$ and for any $s>0$, Lemma 2.5 gives

$$
\left(\int_{0}^{s} P_{\tau}^{(\alpha)} d \tau\right) *\left(u-W^{(\alpha)} \mu_{m}\right) *\left(\tilde{L}^{(\alpha)} \phi\right)^{\sim}(X) \geqq 0,
$$

so that

$$
\int u \phi d X-\int u \cdot\left(\tilde{P}_{s}^{(\alpha)} * \phi\right) d X \geqq \int\left(\int_{0}^{s} \tilde{P}_{\tau}^{(\alpha)} d \tau\right) * \phi d \mu_{m}
$$

Hence

$$
\int u \phi d X \geqq \int W^{(\alpha)} \mu_{m} \phi d X
$$


Thus $u \geqq W^{(\alpha)} \mu_{m}$ a.e. By Lemma $2.4, u \geqq W^{(\alpha)} \mu_{m}$. Letting $m \rightarrow \infty$, we obtain $u \geqq W^{(\alpha)} \mu$. Put

$$
h=u-W^{(\alpha)} \mu \quad \text { on }\left\{X \in R^{n+1} ; W^{(\alpha)} \mu(X)<\infty\right\} .
$$

Then Remark 2.6 gives

$$
\int\left(h-\tilde{P}_{s}^{(\alpha)} * h\right) \phi d X=\left(\int_{0}^{s} P_{\tau}^{(\alpha)} d \tau\right) * h *\left(\tilde{L}^{(\alpha)} \phi\right) \sim(0)=0
$$

for every $s>0$ and $\phi \in C_{K}\left(R^{n+1}\right)$. Hence $h=P_{s}^{(\alpha)} * h$ a.e. For any $\psi \in$ $C_{K}((0, \infty))$ with $\psi \geqq 0$ and with $\int \psi d t=1, h=W_{(\psi)}^{(\alpha)} * h$ a.e. and $\left(W_{(\psi)}^{(\alpha)} * h\right)$ $=P_{s}^{(\alpha)} *\left(W_{(\psi)}^{(\alpha)} * h\right)$ on $R^{n+1}$, so that Lemma 2.8 gives $W_{(\psi)}^{(\alpha)} * h=c$ with some constant $c \geqq 0$, that is, $h=c$ a.e., which gives $u=W^{(\alpha)} \mu+c$ a.e. Lemma 2.4 leads to $u=W^{(\alpha)} \mu+c$, which shows the first equality. By Remark 2.6, we obtain the second part of this proposition. Thus Proposition 2.10 is shown.

Corollary 2.11. Let $u \in S_{\alpha}$ and $\mu \in M_{\alpha}$. If $u \leqq W^{(\alpha)} \mu$, then $u$ is the $W^{(\alpha)}$-potential of the associated measure of $u$.

\section{§3. Reduced functions and swept-out measures}

For $u \in S_{\alpha, c}$ and a compact set $K$ in $R^{n+1}$, we put

$$
Q_{K}^{(\alpha)} u(X)=\inf \left\{v(X) ; v \in S_{\alpha}, v \geqq u \text { on } K\right\}
$$

and

$$
R_{K}^{(\alpha)} u(X)=\underbrace{Q_{K}^{(\alpha)}} u(X),
$$

where $Q_{K}^{(\alpha)} u$ is the lower regularization of $Q_{K}^{(\alpha)} u$, namely for a function $v$ on $R^{n+1}, \underset{v}{v}(X)=\liminf _{Y \rightarrow X} v(Y)$. Furthermore, for $u \in S_{\alpha}$ and a set $A$ in $R^{n+1}$, we put

$$
\begin{gathered}
R_{A}^{(\alpha)} u(X)=\sup \left\{R_{K}^{(\alpha)} v(X) ; v \in S_{\alpha, c}, v \leqq u \text { and } A \supset K: \text { compact set }\right\} \\
\bar{Q}_{A}^{(\alpha)} u(X)=\inf \left\{R_{\omega}^{(\alpha)} u(X) ; A \subset \omega: \text { open set }\right\}
\end{gathered}
$$

and

$$
\bar{R}_{A}^{(\alpha)} u(X)=\underbrace{\bar{Q}_{A}^{(\alpha)}} u(X) .
$$

We say that $R_{A}^{(\alpha)} u$ and $\bar{R}_{A}^{(\alpha)} u$ are the reduced function of $u$ to $A$ and the outer reduced function of $u$ to $A$ with respect to $L^{(\alpha)}$, respectively.

For a set $A$ in $R^{n+1}$ and for $u \in \tilde{S}_{\alpha}$, the reduced function $\tilde{R}_{A}^{(\alpha)} u$ of $u$ to $A$ and the outer reduced function $\overline{\tilde{R}}_{A}^{(\alpha)} u$ of $u$ to $A$ with respect to $\tilde{L}^{(\alpha)}$ 
can be defined analogously. For all the results for $L^{(\alpha)}$ in this paragraph, the analogies for $\tilde{L}^{(\alpha)}$ hold.

Remark 3.1. Let $\omega$ be an open set in $R^{n+1}$ and $u \in S_{\alpha}$. Then we have:

(1) $\omega$ is redusable, that is, $\bar{R}_{\omega}^{(\alpha)} u=R_{\omega}^{(\alpha)} u$.

(2) $R_{\omega}^{(\alpha)} u=u$ on $\omega$.

Lemma 3.2 (G. Choquet, [6] p. 34). Let $\left(f_{i}\right)_{i \in I}$ be an arbitrary family of functions on $R^{n+1}$. Then there exists a countable subset $I_{0}$ of I such that for any lower semi-continuous function $g, g \leqq f_{I_{0}}$ implies $g \leqq f_{I}$. Here for a subset $J$ of $I$, we write $f_{J}(X)=\inf _{i \in J} f_{i}(X)$.

LEMMA 3.3. Let $u$ be a positive and locally integrable Borel function on $R^{n+1}$ and assume $u \geqq P_{s}^{(\alpha)} * u$ for $s>0$. Then $\underset{\sim}{u} \in S_{\alpha}, \underset{u}{u}=u$ a.e. and for any approximate sequence $\left(\phi_{m}\right)_{m=1}^{\infty}$ of the Dirac measure, $\left(W_{\left(\phi_{m}\right)}^{(\alpha)} * u(X)\right)_{m=1}^{\infty}$ converges increasingly to $\underset{u}{u}(X)$ with $m \rightarrow \infty$.

Proof. Take an approximate sequence $\left(\phi_{m}\right)_{m=1}^{\infty}$ of the Dirac measure. The semi-group property of $\left(P_{s}^{(\alpha)}\right)_{s \geqq 0}$ shows that $P_{s_{1}}^{(\alpha)} * u \geqq P_{s_{2}}^{(\alpha)} * u$ on $R^{n+1}$ if $0<s_{1}<s_{2}$, so that $\left(W_{\left(\phi_{m}\right)}^{(\alpha)} * u(X)\right)_{m=1}^{\infty}$ is increasing. For $X \in R^{n+1}$, we choose a sequence $\left(X_{k}\right)_{k=1}^{\infty} \subset R^{n+1}$ convergent to $X$ satisfying $\underset{\sim}{u}(X)=\lim _{k \rightarrow \infty} u\left(X_{k}\right)$. Then for any $m \geqq 1$,

$$
\underline{u}(X) \geqq \liminf _{k \rightarrow \infty}\left(W_{\left(\phi_{m}\right)}^{(\alpha)} * u\left(X_{k}\right)\right) \geqq W_{\left(\phi_{m}\right)}^{(\alpha)} * u(X) \geqq W_{\left(\phi_{m}\right)}^{(\alpha)} * \underset{u}{u}(X) .
$$

For any $\phi \in C_{K}\left(R^{n+1}\right)$ with $\phi \geqq 0$, the Fatou lemma gives

$$
\int u \phi d X \leqq \liminf _{m \rightarrow \infty} \int u \cdot\left(\tilde{W}_{\left(\phi_{m}\right)}^{(\alpha)} * \phi\right) d X=\liminf _{m \rightarrow \infty} \int\left(W_{\left(\phi_{m}\right)}^{(\alpha)} * u\right) \phi d X \leqq \int \underline{u} \phi d X,
$$

so that $u \leqq \underset{\sim}{u}$ a.e., that is, $u=\underset{\sim}{u}$ a.e. Since $\mathrm{w}^{*}-\lim _{m \rightarrow \infty}\left(W_{\left(\phi_{m}\right)}^{(\alpha)} d X\right)=\varepsilon^{3)}$ and $\underline{u}$ is lower semi-continuous, we have

$$
\underset{m \rightarrow \infty}{\liminf }\left(W_{\left(\phi_{m}\right)}^{(\alpha)} * \underset{\sim}{u}(X)\right) \geqq \underset{\sim}{u}(X) \text { on } R^{n+1} .
$$

Thus we have

$$
\underline{u}(X)=\lim _{m \rightarrow \infty}\left(W_{\left(\phi_{m}\right)}^{(\alpha)} * \underset{u}{u}(X)\right)=\lim _{m \rightarrow \infty}\left(W_{\left(\phi_{m}\right)}^{(\alpha)} * u(X)\right) \text { on } R^{n+1} .
$$

This gives $\underset{\sim}{u} \in S_{\alpha}$, which shows Lemma 3.3.

3) For a sequence $\left(\mu_{m}\right)_{m=1}^{\infty}$ of Borel measures and a Borel measure $\mu$, we write $\mu=$ $\mathrm{w}^{*}-\lim _{m \rightarrow \infty} \mu_{m}$ if $\left(\mu_{m}\right)_{m=1}^{\infty}$ converges vaguely to $\mu$ as $m \rightarrow \infty$. 
Lemmas 3.2 and 3.3 give the following

Remark 3.4. For $u \in S_{\alpha}$ and any set $A$ in $R^{n+1}$, we have:

(1) $R_{A}^{(\alpha)} u=\lim _{m \rightarrow \infty} R_{A}^{(\alpha)}\left(W_{\left(\phi_{m}\right)}^{(\alpha)} * u\right), R_{A}^{(\alpha)} u \in S_{\alpha}, \bar{R}_{A}^{(\alpha)} u \in S_{\alpha}$,

(2) $R_{A}^{(\alpha)} u$ is a $W^{(\alpha)}$-potential if $A$ is relatively compact (see Corollary 2.11) and $R_{A}^{(\alpha)} R_{A}^{(\alpha)} u=R_{A}^{(\alpha)} u$ if $A$ is open (see Remark 3.1).

In general, a closed set $F$ is not always reducible, that is, $\bar{R}_{F}^{(\alpha)} u \neq$ $R_{F}^{(\alpha)} u$ for some $u \in S_{\alpha}$. But we have the following

Lemma 3.5. Let $F$ be a closed set in $R^{n+1}$ and $u \in S_{\alpha}$. If $u$ is continuous on a neighborhood of $F$ and if $\lim _{X \in F, X \rightarrow \infty} u(X)=0$, then $\bar{R}_{F}^{(\alpha)} u=R_{F}^{(\alpha)} u$.

Proof. For any $\delta>0$, we choose a compact set $K \subset F$ such that $u \leqq \delta$ on $F \backslash K$. Then we have

$$
R_{K}^{(\alpha)} u \leqq R_{F}^{(\alpha)} u \leqq \bar{R}_{F}^{(\alpha)} u \leqq \bar{R}_{K}^{(\alpha)} u+\delta \text { on } R^{n+1},
$$

so that it suffices to show that $\bar{R}_{K}^{(\alpha)} u=R_{K}^{(\alpha)} u$ for every compact set $K \subset F$. Let $v \in S_{\alpha}$ with $v \geqq u$ on $K$. Then for any $\delta>0$, continuity of $u$ on some neighborhood of $K$ shows that $v+\delta \geqq \bar{R}_{K}^{(\alpha)} u$ on $R^{n+1}$. Letting $\delta \rightarrow 0$ and taking the lower regularizations, we obtain $R_{K}^{(\alpha)} u \geqq \bar{R}_{K}^{(\alpha)} u$ on $R^{n+1}$, that is, $R_{K}^{(\alpha)} u \geqq \bar{R}_{K}^{(\alpha)} u$, which shows Lemma 3.5 .

For $\mu \in M_{\alpha}$ (resp. $\mu \in \tilde{M}_{\alpha}$ ) and for a set $A$ in $R^{n+1}$, Corollary 2.11 shows that $R_{A}^{(\alpha)} W^{(\alpha)} \mu$ (resp. $\tilde{R}_{A}^{(\alpha)} \tilde{W}^{(\alpha)} \mu$ ) is a $W^{(\alpha)}$-potential (resp. $\tilde{W}^{(\alpha)}$-potential). We denote by $\mu_{A}^{\prime}$ (resp. $\mu_{A}^{\prime \prime}$ ) the associated measure of $R_{A}^{(\alpha)} W^{(\alpha)} \mu$ (resp. $\tilde{R}_{A}^{(\alpha)} \tilde{W}^{(\alpha)} \mu$ ). We say that $\mu_{A}^{\prime}$ (resp. $\mu_{A}^{\prime \prime}$ ) is the inner $W^{(\alpha)}$-swept-out (resp. $\tilde{W}^{(\alpha)}$-swept-out) measure of $\mu$ to $A$.

Proposition 3.6. Let $A$ be a set in $R^{n+1}$ and $\mu \in M_{\alpha}$. Then

$$
\int d \mu_{A}^{\prime} \leqq \int d \mu
$$

Proof. Let $\left(\omega_{m}\right)_{m=1}^{\infty}$ be an exhaustion of $R^{n+1}$. By Remark 3.1, there exists a positive measure $\nu_{m}$ with $\tilde{R}_{\omega_{m}}^{(\alpha)} 1=\tilde{W}^{(\alpha)} \nu_{m}$. Then we have

$$
\begin{aligned}
\int d \mu_{A}^{\prime} & =\lim _{m \rightarrow \infty} \int \tilde{W}^{(\alpha)} \nu_{m} d \mu_{\boldsymbol{A}}^{\prime}=\lim _{m \rightarrow \infty} \int W^{(\alpha)} \mu_{\boldsymbol{A}}^{\prime} d \nu_{m} \\
& \leqq \lim _{m \rightarrow \infty} \int W^{(\alpha)} \mu d \nu_{m}=\lim _{m \rightarrow \infty} \int \tilde{W}^{(\alpha)} \nu_{m} d \mu=\int d \mu .
\end{aligned}
$$

Proposition 3.7. Let $u \in S_{\alpha}$ and let $A$ be a set in $R^{n+1}$. Then the support of associated measure of $R_{A}^{(\alpha)} u$ is in $\bar{A}$. 
Proof. By the definition of $R_{A}^{(\alpha)} u$, Lemma 2.7 and by Remarks 3.5, 3.4, we may assume that $A$ is compact and that $u$ is a continuous $W^{(a)}$ potential. Put $u=W^{(\alpha)} \mu$ with $\mu \in M_{\alpha}$ and let $\left(\omega_{m}\right)_{m=1}^{\infty}$ be a sequence of relatively compact open sets with $\bar{\omega}_{m+1} \subset \omega_{m}$ and with $\bigcap_{m=1}^{\infty} \omega_{m}=A$. Since $W^{(\alpha)} \mu_{\omega_{m}}^{\prime} \leqq W^{(\alpha)} \mu$ for all $m$ and since Lemmas 3.3 and 3.5 give $\lim _{m \rightarrow \infty}\left(W^{(\alpha)} \mu_{\omega_{m}}^{\prime}\right)$ $=W^{(\alpha)} \mu_{A}^{\prime}$ a.e., we obtain $\mu_{A}^{\prime}=\mathrm{w}^{*}-\lim _{m \rightarrow \infty} \mu_{\omega_{m}}^{\prime}$ (see Lemma 2.7). Hence it suffices to show $\operatorname{supp}\left[\mu_{\omega}^{\prime}\right] \subset \bar{\omega}$ for every open set $\omega$ in $R^{n+1}$. Suppose that there exists a point $X_{0} \in C \bar{\omega} \cap \operatorname{supp}\left[\mu_{\omega}^{\prime}\right]$. Let $\left(V_{m}\right)_{m=1}^{\infty}$ be a sequence of open sets in $R^{n+1}$ with $\bar{V}_{1} \subset C \bar{\omega}, \overline{V_{m+1}} \subset V_{m}$ and with $\bigcap_{m=1}^{\infty} V_{m}=\left\{X_{0}\right\}$. We put $\mu_{m}=\left.\mu_{\omega}^{\prime}\right|_{V_{m}}$. Then

$$
W^{(\alpha)} \mu_{\omega}^{\prime} \geqq W^{(\alpha)}\left(\mu_{\omega}^{\prime}-\mu_{m}\right)+W^{(\alpha)}\left(\mu_{m}\right)_{\omega}^{\prime} \quad \text { on } R^{n+1}
$$

and

Hence

$$
W^{(\alpha)}\left(\mu_{\omega}^{\prime}-\mu_{m}\right)+W^{(\alpha)}\left(\mu_{m}\right)_{\omega}^{\prime}=W^{(\alpha)} \mu \quad \text { on } \omega \text {. }
$$

so that

$$
W^{(\alpha)} \mu_{m}=W^{(\alpha)}\left(\mu_{m}\right)_{\omega}^{\prime} \quad \text { on } R^{n+1}
$$

$$
\lim _{m \rightarrow \infty} W^{(\alpha)}\left(\left(\mu_{m}\right)_{\omega}^{\prime} / \int d \mu_{m}\right)=\lim _{m \rightarrow \infty} W^{(\alpha)}\left(\mu_{m} / \int d \mu_{m}\right)=W^{(\alpha)} \varepsilon_{X_{0}} \quad \text { on } C\left\{X_{0}\right\},
$$

which contradicts the unboundednes s of $W^{(\alpha)} \varepsilon_{X_{0}}$ on a neighborhood of $X_{0}$. Thus Proposition 3.7 is shown.

Proposition 3.8. Let $\mu \in M_{\alpha}$ and $\nu \in \tilde{M}_{\alpha}$. For a set $A$ in $R^{n+1}$, we have

$$
\int W^{(\alpha)} \mu_{A}^{\prime} d \nu=\int W^{(\alpha)} \mu d \nu_{A}^{\prime \prime} \quad \text { and } \quad W^{(\alpha)} \mu_{A}^{\prime}(X)=\int W^{(\alpha)} \varepsilon_{Y, A}^{\prime}(X) d \mu(Y),
$$

where we denote by $\varepsilon_{Y}$ and by $\varepsilon_{Y,{ }_{A}}^{\prime}$ the Dirac measure at $Y$ and its inner $W^{(\alpha)}$-swept-out measure to $A$. In particular if $A$ is open,

$$
\int W^{(\alpha)} \mu_{A}^{\prime} d \nu=\int W^{(\alpha)} \mu_{A}^{\prime} d \nu_{A}^{\prime \prime}
$$

Proof. First we assume that $A$ is open. Let $\left(\omega_{m}\right)_{m=1}^{\infty}$ be an exhaustion of $A$. Then Proposition 3.7 and Remark 3.1 show that

$$
\begin{aligned}
\int W^{(\alpha)} \mu_{A}^{\prime} d \nu & =\lim _{m \rightarrow \infty} \int W^{(\alpha)} \mu_{\omega_{m}}^{\prime} d \nu=\lim _{m \rightarrow \infty} \int \tilde{W}^{(\alpha)} \nu d \mu_{\omega_{m}}^{\prime}=\lim _{m \rightarrow \infty} \int \tilde{W}^{(\alpha)} \nu_{A}^{\prime \prime} d \mu_{\omega_{m}}^{\prime} \\
& =\lim _{m \rightarrow \infty} \int W^{(\alpha)} \mu_{\omega_{m}}^{\prime} d \nu_{A}^{\prime \prime}=\int W^{(\alpha)} \mu_{A}^{\prime} d \nu_{A}^{\prime \prime} .
\end{aligned}
$$

Let $A$ be an ar bitrary set. By the definition of inner $W^{(\alpha)}$-swept-out 
measures and Lemma 3.3, we may assume that $A$ is compact, $\mu \in M_{\alpha, c}$ and that $\nu \in \tilde{M}_{\alpha, c}$. Take a sequence $\left(\Omega_{m}\right)_{m=1}^{\infty}$ of relatively compact open sets with $\overline{\Omega_{m+1}} \subset \Omega_{m}$ and with $\bigcap_{m=1}^{\infty} \Omega_{m}=A$. By Lemma 3.5 and the above result, we have

$$
\int W^{(\alpha)} \mu_{A}^{\prime} d \nu=\lim _{m \rightarrow \infty} \int W^{(\alpha)} \mu_{\Omega_{m}}^{\prime} d \nu=\lim _{m \rightarrow \infty} \int \tilde{W}^{(\alpha)} \nu_{\Omega_{m}}^{\prime \prime} d \mu=\int \tilde{W}^{(\alpha)} \nu_{A}^{\prime \prime} d \mu .
$$

In particular, we have $W^{(\alpha)} \varepsilon_{Y, A}^{\prime}(X)=\tilde{W}^{(\alpha)} \varepsilon_{X,{ }_{A}}^{\prime \prime}(Y)$. Hence

$$
W^{(\alpha)} \mu_{A}^{\prime}(X)=\int W^{(\alpha)} \mu_{A}^{\prime} d \varepsilon_{X}=\int \tilde{W}^{(\alpha)} \varepsilon_{X, A}^{\prime \prime}(Y) d \mu(Y)=\int W^{(\alpha)} \varepsilon_{Y, A}^{\prime}(X) d \mu(Y) .
$$

This completes the proof.

By Remark 3.4, (2) and Proposition 3.8, we have the following

CoRollary 3.9. Let $\omega$ be an open set in $R^{n+1}$. Then the mapping $M_{\alpha} \ni \mu \rightarrow \mu_{\omega}^{\prime}$ is positively linear, and for any $\mu \in M_{\alpha}$ and any positive measure $\nu$ with $\nu \leqq \mu_{\omega}^{\prime}$, we have $\nu_{\omega}^{\prime}=\nu$.

Proof. It follows immediately from Proposition 3.8 that the mapping $\mu \rightarrow \mu_{\omega}^{\prime}$ is positively linear. By Remark 3.4 , (2) we have $\left(\mu_{\omega}^{\prime}\right)_{\omega}^{\prime}=\mu_{\omega}^{\prime}$, so that by Proposition 3.8 , for any $X \in R^{n+1}$,

$$
\int\left(W^{(\alpha)} \varepsilon_{Y}(X)-W^{(\alpha)} \varepsilon_{Y, \omega}^{\prime}(X)\right) d \mu_{\omega}^{\prime}(Y)=0 .
$$

Since $W^{(\alpha)} \varepsilon_{Y} \geqq W^{(\alpha)} \varepsilon_{Y, \omega}^{\prime}$, we have $W^{(\alpha)} \varepsilon_{Y}=W^{(\alpha)} \varepsilon_{Y, \omega}^{\prime} \mu_{\omega}^{\prime}$-a.e. as functions of $Y$, so that

$$
\int\left(W^{(\alpha)} \varepsilon_{Y}(X)-W^{(\alpha)} \varepsilon_{Y, \omega}^{\prime}(X)\right) d \nu(Y)=0,
$$

that is,

$$
W^{(\alpha)} \nu=W^{(\alpha)} \nu_{\omega}^{\prime},
$$

which gives $\nu=\nu_{\omega}^{\prime}$.

Proposition 3.10. Let $\mu \in M_{\alpha}$. Then we have:

(1) For two sets $A_{1}$ and $A_{2}$ in $R^{n+1}$ with $A_{1} \subset A_{2}$, we have $\mu_{A_{1}}^{\prime} \geqq \mu_{A_{2}}^{\prime}$ on $\operatorname{Int}\left(A_{1}\right)$, where $\operatorname{Int}\left(A_{1}\right)$ denotes the interior of $A_{1}$.

(2) For a set $A$ in $R^{n+1}$ with $\int_{\overline{C A}} d \mu=0$, we have $\mu_{A}^{\prime}=\mu$.

Proof. (1): Choose $\phi \in C_{K}^{\infty}\left(R^{n+1}\right)$ with $\phi \geqq 0$ and $\operatorname{supp}[\phi] \subset \operatorname{Int}\left(A_{1}\right)$. Let $\lambda$ be the real Borel measure such that $\phi=\tilde{W}^{(\alpha)} \lambda$. Then we have 


$$
\begin{aligned}
\int \tilde{W}^{(\alpha)} \lambda d \mu_{A_{2}}^{\prime} & =\int W^{(\alpha)} \mu_{A_{2}}^{\prime} d \lambda^{+}-\int W^{(\alpha)} \mu_{A_{2}}^{\prime} d \lambda^{-} \\
& \leqq \int W^{(\alpha)} \mu_{A_{1}}^{\prime} d \lambda^{+}-\int W^{(\alpha)} \mu_{A_{1}}^{\prime} d \lambda^{-} \\
& =\int \tilde{W}^{(\alpha)} \lambda d \mu_{A_{1}}^{\prime},
\end{aligned}
$$

because $\operatorname{supp}\left[\lambda^{+}\right] \subset \operatorname{Int}\left(A_{1}\right)$ and $W^{(\alpha)} \mu_{A_{1}}^{\prime}=W^{(\alpha)} \mu_{A_{2}}^{\prime}$ on $\operatorname{Int}\left(A_{1}\right)$.

By using Proposition 3.8 and Remark 3.1, (2), we show (2) in the same manner as in (1). This completes the proof.

Proposition 3.11 (the domination principle). Let $\Omega$ be an open set in $R^{n+1}, u \in S_{\alpha}$ and $\mu \in M_{\alpha}$ with $\operatorname{supp}[\mu] \subset \Omega . \quad$ Put

$$
E=\left\{X \in \Omega ; u(X)-R_{C \Omega}^{(\alpha)} u(X) \geqq W^{(\alpha)} \mu(X)-W^{(a)} \mu_{C \Omega}^{\prime}(X)\right\} .
$$

If $\mu_{E}^{\prime}=\mu$, then $u-R_{C \Omega}^{(\alpha)} u \geqq W^{(\alpha)} \mu-W^{(\alpha)} \mu_{C \Omega}^{\prime}$ on $R^{n+1}$.

Proof. Since $\mu$ is a sum of positive measures with compact support, we may assume that $\operatorname{supp}[\mu]$ is compact. Let $\omega$ be an open set with $\omega \supset C \Omega$. Then

$$
u+R_{\omega}^{(\alpha)} W^{(\alpha)} \mu \geqq R_{C \Omega}^{(\alpha)} u+W^{(\alpha)} \mu \text { on } E \cup \omega .
$$

Let $\nu$ be the associated measure of $R_{C \Omega}^{(\alpha)} u$ and put $R_{C \Omega}^{(\alpha)} u=W^{(\alpha)} \nu+c$ with $c \geqq 0, \quad$ Then $\operatorname{supp}[\nu] \subset C \Omega$, so that

$$
R_{C \Omega}^{(\alpha)} u+W^{(\alpha)} \mu=c+R_{E \cup \omega}^{(\alpha)}\left(W^{(\alpha)} \nu+W^{(\alpha)} \mu\right) \leqq u+R_{\omega}^{(\alpha)} W^{(\alpha)} \mu \text { on } R^{n+1},
$$

because $u-c+R_{\omega}^{(\alpha)} W^{(\alpha)} \mu \geqq 0$. Since $W^{(\alpha)} \mu$ is continuous in a certain neighborhood of $C \Omega$ and vanishes at the infinity, Lemma 3.5 shows

$$
u-R_{C \Omega}^{(\alpha)} u \geqq W^{(\alpha)} \mu-W^{(\alpha)} \mu_{C \Omega}^{\prime},
$$

which shows Proposition 3.11.

Proposition 3.12. Let $\omega_{1}$ and $\omega_{1}$ be open sets in $R^{n+1}$ with $\overline{\omega_{1}} \cap \overline{\omega_{2}}=\phi$ and $\mu \in M_{\alpha}$. Then $\mu_{\omega_{1}}^{\prime}=\mu_{\omega_{1} \cup \omega_{2}}^{\prime} \mid \overline{\omega_{1}}+\left(\mu_{\omega_{1} \cup \omega_{2}}^{\prime} \mid \overline{\omega_{2}}\right)_{\omega_{1}}^{\prime}$.

Proof. Let $\left(\omega_{1, m}\right)_{m=1}^{\infty}$ be an exhaustion of $\omega_{1}$ and put $\mu_{1}=\left.\mu_{\omega_{1} \cup \omega_{2}}^{\prime}\right|_{\overline{\omega_{1}}}$ and $\mu_{m}^{\prime}=\left(\mu_{1}\right)_{\omega_{1}, m \cup \omega_{2}}^{\prime}$. Since $\operatorname{supp}\left[\left.\mu_{m}^{\prime}\right|_{\omega_{1}}\right] \subset \overline{\omega_{1, m}} \subset \omega_{1}$, by Proposition 3.10, (2), $\left.\mu_{m}^{\prime}\right|_{\omega_{1}}$ $=\left(\left.\mu_{m}^{\prime}\right|_{\omega_{1}}\right)_{\omega_{1}}^{\prime}$, so that

$$
W^{(\alpha)}\left(\left.\mu_{m}^{\prime}\right|_{\omega_{1}}\right)=R_{\omega_{1}}^{(\alpha)} W^{(\alpha)}\left(\left.\mu_{m}^{\prime}\right|_{\omega_{1}}\right) \leqq R_{\omega_{1}}^{(\alpha)} W^{(\alpha)} \mu_{1}=W^{(\alpha)}\left(\mu_{1}\right)_{\omega_{1}}^{\prime} .
$$

On the other hand, by Corollary 3.9 , we have $\left(\mu_{1}\right)_{\omega_{1} \cup \omega_{2}}^{\prime}=\mu_{1}$, so that 
$\left.\left(\mu_{1}\right)_{\omega_{1} \cup \omega_{2}}^{\prime}\right|_{\omega_{1}}=\mu_{1}$. Since $\mathrm{w}^{*}-\lim _{m \rightarrow \infty} \mu_{m}^{\prime}=\left(\mu_{1}\right)_{\omega_{1} \cup \omega_{2}}^{\prime}$ by Lemma 2.7 , it follows that

$$
W^{(\alpha)} \mu_{1}=W^{(\alpha)}\left(\left.\left(\mu_{1}\right)_{\omega_{1} \cup \omega_{2}}^{\prime}\right|_{\overline{\omega_{1}}}\right) \leqq \liminf _{m \rightarrow \infty} W^{(\alpha)}\left(\left.\mu_{m}^{\prime}\right|_{\omega_{1}}\right) \leqq W^{(\alpha)}\left(\mu_{1}\right)_{\omega_{1}}^{\prime} .
$$

Thus, $\mu_{1}=\left(\mu_{1}\right)_{\omega_{1}}^{\prime}$, and hence $\mu_{\omega_{1}}^{\prime}=\left(\mu_{\omega_{1} \cup \omega_{2}}^{\prime}\right)_{\omega_{1}}^{\prime}=\mu_{1}+\left(\mu_{\omega_{1} \cup \omega_{2}}^{\prime} \frac{1}{\omega_{\omega_{2}}}\right)_{\omega_{1}}^{\prime}$, which shows Proposition 3.12 .

Corollary 3.13. Let $\Omega$ and $\omega$ be open sets in $R^{n+1}$ and $\mu \in M_{\alpha}$. Then $\left(\left.\mu_{\Omega}^{\prime}\right|_{\omega}\right)_{\Omega \cap \omega}^{\prime}=\left.\mu_{\Omega}^{\prime}\right|_{\omega}$.

Proof. By Proposition 3.8, we may assume that supp [ $\mu]$ is compact. Let $\left(\omega_{m}\right)_{m=1}^{\infty}$ be an exhaustion of $\omega$. By Proposition 3.6, we may assume that $\left(\left(\left.\mu_{\Omega}^{\prime}\right|_{\omega}\right)_{\Omega \cap\left(\omega_{m} \cup \overline{\omega_{m+1}}\right)}^{\prime}\right)_{m=1}^{\infty}$ converges vaguely to some measure $\nu$. By the definition of inner $W^{(\alpha)}$-swept-out measures, we have $\nu=\left(\left.\mu_{\Omega}^{\prime}\right|_{\omega}\right)_{\Omega}^{\prime}=\left.\mu_{\Omega}^{\prime}\right|_{\omega}$ (see Corollary 3.9). Proposition 3.12 gives

$$
\left.\left(\left.\mu_{\Omega}^{\prime}\right|_{\omega}\right)_{\Omega \cap\left(\omega_{m} \cup \overline{\omega_{m}+1}\right.}^{\prime}\right) \leqq\left(\left.\mu_{\Omega}^{\prime}\right|_{\omega}\right)_{\Omega \cap \omega_{m}}^{\prime} \quad \text { on } \omega_{m} .
$$

Letting $m \rightarrow \infty$, we have

$$
\left.\mu_{\Omega}^{\prime}\right|_{\omega} \leqq\left(\left.\mu_{\Omega}^{\prime}\right|_{\omega}\right)_{\Omega \cap \omega}^{\prime} \quad \text { on } \omega \text {. }
$$

Hence Proposition 3.6 shows $\left.\mu_{\Omega}^{\prime}\right|_{\omega}=\left(\left.\mu_{\Omega}^{\prime}\right|_{\omega}\right)_{\Omega \cap \omega}^{\prime}$.

Proposition 3.14. Let $\Omega$ be an open set in $R^{n+1}, T$ the projection of $C \bar{\Omega}$ to the $t$-axis and $X_{0}=\left(x_{0}, t_{0}\right) \in R^{n+1}$. Let $M$ be the connected component of $T \cup\left\{t_{0}\right\}$ satisfying $t_{0} \in M$ and put $t_{1}=\sup M$. If $\varepsilon_{X_{0}, \Omega}^{\prime} \neq \varepsilon_{X_{0}}$, then

$$
\operatorname{supp}\left[\varepsilon_{X_{0}, \Omega}^{\prime}\right] \supset \bar{\Omega} \cap\left(R^{n} \times\left(t_{0}, t_{1}\right)\right) .
$$

For the proof, we use the following

Lemma 3.15. Let $\mu, \nu \in M_{\alpha}$ and $X_{0}=\left(x_{0}, t_{0}\right) \in R^{n+1} \backslash \operatorname{supp}[\nu]$. Suppose that $W^{(\alpha)} \mu \geqq W^{(\alpha)} \nu$ on $R^{n+1}$ and that $\operatorname{supp}[\mu] \subset\left\{(x, t) \in R^{n+1} ; t<t_{0}\right\}$. If $W^{(\alpha)} \mu\left(X_{0}\right)=W^{(\alpha)} \nu\left(X_{0}\right)$, then $\operatorname{supp}[\nu] \subset\left\{(x, t) \in R^{n+1} ; t \leqq t_{0}\right\}$ and $W^{(\alpha)} \mu=W^{(\alpha)} \nu$ on $\left\{(x, t) \in R^{n+1} ; t>t_{0}\right\}$.

Proof. Since $W^{(\alpha)}(\mu-\nu) \geqq 0, W^{(\alpha)}(\mu-\nu)\left(X_{0}\right)=0$ and since $W^{(\alpha)}(\mu-\nu)$ is of class $C^{\infty}$ in a neighborhood of $X_{0}$,

$$
W^{(\alpha)}(\mu-\nu)\left(X_{0}\right)=\frac{\partial}{\partial t} W^{(\alpha)}(\mu-\nu)\left(X_{0}\right)=0
$$

and

$$
0=L^{(\alpha)} W^{(\alpha)}(\mu-\nu)\left(X_{0}\right)=-C_{n, \alpha} \int_{R^{n}} W^{(\alpha)}(\mu-\nu)\left(x_{0}-y, t_{0}\right)|y|^{-n-2 \alpha} d y
$$


Then we have $W^{(\alpha)} \mu\left(x, t_{0}\right)=W^{(\alpha)} \nu\left(x, t_{0}\right) d x$-a.e., so that for any $s>0$ and for any $x \in R^{n}$, we have

$$
\begin{aligned}
W^{(\alpha)} \mu\left(x, t_{0}+s\right) & =\int W^{(\alpha)}(x-y, s) W^{(\alpha)} \mu\left(y, t_{0}\right) d y \\
& =\int W^{(\alpha)}(x-y, s) W^{(\alpha)} \nu\left(y, t_{0}\right) d y \\
& \leqq W^{(\alpha)} \nu\left(x, t_{0}+s\right) .
\end{aligned}
$$

Therefore $W^{(\alpha)} \mu=W^{(\alpha)} \nu$ on $\left\{(x, t) \in R^{n+1} ; t>t_{0}\right\}$ and $\nu=0$ on $\left\{(x, t) \in R^{n+1}\right.$; $\left.t>t_{0}\right\}$, which shows Lemma 3.15 .

Proof of Proposition 3.14. Put

$$
s=\sup \left\{t \geqq t_{0} ; \operatorname{supp}\left[\varepsilon_{X_{0}, \Omega}^{\prime}\right] \cap\left(R^{n} \times\{t\}\right) \neq \phi\right\} .
$$

Then Lemma 3.15 yields $s>t_{0}$ and

$$
\operatorname{supp}\left[\varepsilon_{X_{0}, \Omega}^{\prime}\right]=\overline{\Omega \cap\left(R^{n} \times\left(t_{0}, s\right)\right)} .
$$

Suppose that $s<t_{1}$ and $\Omega \cap\left(R^{n} \times(s, \infty)\right) \neq \phi$; we can take a nonempty open set $\omega$ in $R^{n}$ and a positive number $\delta>0$ such that $t_{0}<s-\delta$ and

$$
D_{\delta}=\omega \times(s-\delta, s) \subset C \bar{\Omega} .
$$

Put $\nu_{\delta}=\varepsilon_{X_{0}, \Omega \cup D_{\delta}}^{\prime} \mid \overline{D_{\tilde{\delta}}}$. If $\nu_{\bar{o}}=0$, then Proposition 3.12 gives $\varepsilon_{X_{0}, \Omega}^{\prime}=\varepsilon_{X_{0}, \Omega \cup D_{\delta}}^{\prime}$, so that Lemma 3.15 shows that $\varepsilon_{X_{0}, \Omega}^{\prime}$ vanishes on $R^{n} \times(s-\delta, \infty)$, which is a contradiction. Hence $\nu_{\hat{o}} \neq 0$ for every sufficiently small $\delta>0$. By Lemma 3.15, there exists $s^{\prime}>s$ such that

$$
W^{(\alpha)} \nu_{\delta}=W^{(\alpha)} \nu_{j, \Omega}^{\prime} \quad \text { on } R^{n} \times\left[s^{\prime}, \infty\right) .
$$

Since Proposition 3.12 shows $\operatorname{supp}\left[\nu_{\delta}+\nu_{\delta, \Omega}^{\prime}\right] \subset R^{n} \times(-\infty, s]$, for any $s<t<s^{\prime}$, we have

$$
\begin{aligned}
0 & =W^{(\alpha)} \nu_{\delta}\left(0, s^{\prime}\right)-W^{(\alpha)} \nu_{\hat{\delta}, \Omega}^{\prime}\left(0, s^{\prime}\right) \\
& =\int_{R^{n}}\left(W^{(\alpha)} \nu_{\hat{\delta}}(x, t)-W^{(\alpha)} \nu_{\hat{\delta}, \Omega}^{\prime}(x, t)\right) W^{(\alpha)}\left(-x, s^{\prime}-t\right) d x .
\end{aligned}
$$

Since $W^{(\alpha)} \nu_{\delta} \geqq W^{(\alpha)} \nu_{\delta, \Omega}^{\prime}$ on $R^{n+1}$, Lemma 2.4 , (3) shows

$$
W^{(\alpha)} \nu_{\delta} \geqq W^{(\alpha)} \nu_{\delta, \Omega}^{\prime} \quad \text { on } R^{n} \times(s, \infty) \text {. }
$$

We may assume that $\left(\nu_{\delta} / \int d \nu_{\delta}\right)_{\delta>0}$ and $\left(\nu_{\delta, \Omega}^{\prime} / \int d \nu_{\delta}\right)_{\delta>0}$ converges vaguely as $\delta \rightarrow 0$. Put 


$$
\nu=\mathrm{w}_{\delta \downarrow 0}^{*} \lim \left(\nu_{\delta} / \int d \nu_{\delta}\right) \quad \text { and } \quad \nu^{\prime}=\mathrm{w}_{\delta \downarrow}^{*}-\lim \left(\nu_{\delta, \Omega}^{\prime} / \int d \nu_{\delta}\right)
$$

then Proposition 3.12 gives $\operatorname{supp}\left[\nu^{\prime}\right] \subset \bar{\Omega} \cap\left(R^{n} \times\left[t_{0}, s\right]\right), W^{(\alpha)} \nu \geqq W^{(\alpha)} \nu^{\prime}$ on

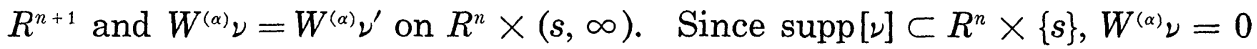
on $R^{n} \times(-\infty, s]$. Hence $W^{(\alpha)} \nu=W^{(\alpha)} \nu^{\prime}$ on $R^{n+1}$, which implies $\nu=\nu^{\prime}$. But this contradicts supp $[\nu] \subset C \bar{\Omega}$ and $\operatorname{supp}\left[\nu^{\prime}\right] \subset \bar{\Omega}$. Thus Proposition 3.14 is shown.

\section{§4. $L^{(\alpha)}$-regular points and a Poincaré type condition}

As in the classical potential theory, we define $L^{(\alpha)}$-regular points for Dirichlet problem.

Definition 2. Let $\Omega$ be an open set in $R^{n+1}$ and $X_{0} \in \partial \Omega$. Then $X_{\mathrm{v}}$ is said to be regular for the Dirichlet problem of $L^{(\alpha)}$ on $\Omega$ if

$$
\mathrm{w}_{X \in \Omega, X \rightarrow X_{0}}^{*} \lim _{X, C \Omega}=\varepsilon_{X_{0}} .
$$

Proposition 4.1. Let $\Omega$ and $\Omega^{\prime}$ be open sets in $R^{n+1}$ and $X_{0} \in \partial \Omega \cap \partial \Omega^{\prime}$. If there exists a neighborhood $V$ of $X_{0}$ such that $\Omega \cap V=\Omega^{\prime} \cap V$ and if $X_{0}$ is regular for the Dirichlet problem of $L^{(\alpha)}$ on $\Omega^{\prime}$, then $X_{0}$ is so on $\Omega$.

Proof. Let $U$ be an open neighborhood of $X_{0}$ with $\bar{U} \subset V$. Then $\mathrm{w}^{*}-\lim _{X \in \Omega, X \rightarrow X_{0}} \varepsilon_{X}^{\prime \prime},\left.C \Omega^{\prime}\right|_{U}=\varepsilon_{X_{0}}$. For any $X \in \Omega$, Lemma 3.5 and the domination principle of $\tilde{W}^{(\alpha)}$ (Proposition 3.11) show

$$
\tilde{W}^{(\alpha)}\left(\left.\varepsilon_{X, C \Omega^{\prime}}^{\prime \prime}\right|_{U}\right) \leqq \overline{\tilde{R}}_{C \Omega}^{(\alpha)} \tilde{W}^{(\alpha)} \varepsilon_{X}=\tilde{R}_{C \Omega}^{(\alpha)} \tilde{W}^{(\alpha)} \varepsilon_{X}=\tilde{W}^{(\alpha)}\left(\varepsilon_{X, C \Omega}^{\prime \prime}\right) \leqq \tilde{W}^{(\alpha)} \varepsilon_{X} .
$$

Let $\left(X_{m}\right)_{m=1}^{\infty}$ be an arbitrary sequence in $\Omega$ with $\lim _{m \rightarrow \infty} X_{m}=X_{0}$. Since $\int d \varepsilon_{X_{m}, C \Omega}^{\prime \prime} \leqq 1$, it suffices to show $\mathrm{w}^{*}-\lim _{m \rightarrow \infty} \varepsilon_{X_{m}, C \Omega}^{\prime \prime}=\varepsilon_{X_{0}}$ in the case that $\left(\varepsilon_{X_{m}, C \Omega}^{\prime \prime}\right)_{m=1}^{\infty}$ converges vaguely. Put $\mu=\mathrm{w}^{*}-\lim _{m \rightarrow \infty} \varepsilon_{X_{m}, C \Omega}^{\prime \prime}$. Since for any non-negative $f \in C_{K}\left(R^{n+1}\right), W^{(\alpha)}(f d X)$ is finite continuous and vanishes at the infinity,

$$
\begin{aligned}
\int \tilde{W}^{(\alpha)} \varepsilon_{X_{0}} f d X & =\lim _{m \rightarrow \infty} \int \tilde{W}^{(\alpha)}\left(\left.\varepsilon_{X_{m}, c \Omega^{\prime}}^{\prime \prime}\right|_{U}\right) f d X \\
& \leqq \lim _{m \rightarrow \infty} \int \tilde{W}^{(\alpha)}\left(\varepsilon_{X_{m}, C \Omega}^{\prime \prime}\right) f d X=\int \tilde{W}^{(\alpha)} \mu \cdot f d X \\
& \leqq \int \tilde{W}^{(\alpha)} \varepsilon_{X_{0}} f d X .
\end{aligned}
$$

Therefore $\tilde{W}^{(\alpha)} \varepsilon_{X_{0}}=\tilde{W}^{(\alpha)} \mu$ a.e., so that $\tilde{W}^{(\alpha)} \varepsilon_{X_{0}}=\tilde{W}^{(\alpha)} \mu$, which gives $\mu=\varepsilon_{X_{0}}$. This shows that $X_{0}$ is regular for the Dirichlet problem of $L^{(\alpha)}$ on $\Omega$. 
Proposition 4.2. Let $\Omega$ be an open set in $R^{n+1}$ and $X_{0}=\left(x_{0}, t_{0}\right) \in \partial \Omega$ such that for any neighborhood $V$ of $X_{0}$,

$$
V \cap \Omega \cap\left\{(x, t) ; t<t_{0}\right\} \neq \phi .
$$

Then the following four conditions are equivalent:

(1) $X_{0}$ is regular for the Dirichlet problem of $L^{(\alpha)}$ on $\Omega$.

(2) For any $u \in S_{\alpha}, R_{C \Omega}^{(\alpha)} u\left(X_{0}\right)=u\left(X_{0}\right)$.

(3) There exist $u \in S_{\alpha}$ and $\left\{\left(x_{m}, t_{m}\right)\right\}_{m=1}^{\infty} \subset R^{n+1}$ such that $t_{m}<t_{0}$, $\lim _{m \rightarrow \infty} t_{m}=t_{0}, R_{C \Omega}^{(\alpha)} u\left(x_{m}, t_{m}\right) \neq u\left(x_{m}, t_{m}\right)$ and that $R_{C \Omega}^{(\alpha)} u\left(X_{0}\right)=u\left(X_{0}\right)$.

(4) $\varepsilon_{X_{0}, c \Omega}^{\prime \prime}=\varepsilon_{X_{0}}$.

Proof. Proposition 3.8 shows for any $\mu \in M_{\alpha}, \quad R_{C \Omega}^{(\alpha)} W^{(\alpha)} \mu\left(X_{0}\right)=$ $\int W^{(\alpha)} \mu d \varepsilon_{X_{0}, C \Omega}^{\prime \prime}$, so that $(2) \leftrightarrow(4)$ holds.

(1) $\rightarrow(3)$ : Choose $f \in C_{K}\left(R^{n+1}\right)$ such that $f \geqq 0$ and that $f>0$ on a neighborhood of $X_{0}$. Then $W^{(\alpha)}(f d X)$ is a required function. In fact, since $W^{(\alpha)}(f d X)$ is finite continuous and vanishes at the infinity, we have

$$
\lim _{Y \in \Omega, Y \rightarrow X_{0}} \int W^{(\alpha)}(f d X) d \varepsilon_{Y, c \Omega}^{\prime \prime}=W^{(\alpha)}(f d X)\left(X_{0}\right),
$$

so that Proposition 3.8 gives

$$
\lim _{Y \in \Omega, Y \rightarrow X_{0}} R_{C \Omega}^{(\alpha)} W^{(\alpha)}(f d X)(Y)=W^{(\alpha)}(f d X)\left(X_{0}\right) .
$$

Since

$$
\mathbf{Q}_{C \Omega}^{(\alpha)} W^{(\alpha)}(f d X)(Y)=W^{(\alpha)}(f d X)(Y) \quad \text { on } C \Omega,
$$

we have

$$
R_{C 2}^{(\alpha)} W^{(\alpha)}(f d X)\left(X_{0}\right)=W^{(\alpha)}(f d X)\left(X_{0}\right) .
$$

Assume $W^{(\alpha)}(f d X)=R_{C \Omega}^{(\alpha)} W^{(\alpha)}(f d X)$ on $R^{n} \times\left(t, t_{0}\right)$ with some $t<t_{0}$ and denote by $f_{1}$ the restriction of $f$ to $R^{n} \times\left(t, t_{0}\right)$. Then Propositions 3.8, 3.11 show

$$
W^{(\alpha)}\left(f_{1} d X\right)=R_{C \Omega}^{(\alpha)} W^{(\alpha)}\left(f_{1} d X\right) \quad \text { on } R^{n+1},
$$

which contradicts Proposition 2.10. Thus (3) holds.

$(3) \rightarrow(4)$ : By Proposition 3.7, $u-R_{C \Omega}^{(\alpha)} u$ is lower semi-continuous on $\Omega$. Furthermore for any $\delta>0$,

$$
\left\{X \in \Omega ; u(X)>R_{C \Omega}^{(\alpha)} u(X)\right\} \cap\left(R^{n} \times\left(t_{0}-\delta, t_{0}\right)\right) \neq \phi .
$$

In fact, if $u(X)=R_{c .2}^{(\alpha)} u(X)$ on $\Omega \cap\left(R^{n} \times\left(t_{0}-\delta, t_{0}\right)\right), \quad L^{(\alpha)} u=0$ on $\Omega \cap$ 
$\left(R^{n} \times\left(t_{0}-\delta, t_{0}\right)\right)$ (in the sense of distributions), because $L^{(\alpha)}\left(R_{C Q}^{(\alpha)} u\right)=0$ on $\Omega$, and hence for any $(x, t) \in \Omega \cap\left(R^{n} \times\left(t_{0}-\delta, t_{0}\right)\right)$,

$$
\int_{R^{n}}\left(u-R_{C \Omega}^{(\alpha)} u\right)(x+y, t)|y|^{-n-2 \alpha} d y=0,
$$

that is, $u=R_{C \Omega}^{(\alpha)} u$ on $R^{n} \times\left(t_{0}-\delta, t_{0}\right)$ (see Lemma 2.4, (3)), which contradicts (3). Hence we can choose $\mu_{\delta} \in M_{\alpha}$ such that $\mu_{\delta} \neq 0, \operatorname{supp}\left[\mu_{\delta}\right] \subset \Omega \cap$ $\left(R^{n} \times\left(t_{0}-\delta, t_{0}\right)\right)$ and that $u-R_{C \Omega}^{(\alpha)} u \geqq W^{(\alpha)} \mu_{\delta}$ on a certain neighborhood of $\operatorname{supp}\left[\mu_{\delta}\right]$. Then Proposition 3.11 gives

$$
u-R_{C \Omega}^{(\alpha)} u \geqq W^{(\alpha)} \mu_{\delta}-W^{(\alpha)} \mu_{\delta, C \Omega}^{\prime} \quad \text { on } R^{n+1},
$$

so that by Proposition 3.8, and the assumption that $u\left(X_{0}\right)=R_{C \Omega}^{(\alpha)} u\left(X_{0}\right)$,

$$
\tilde{W}^{(\alpha)} \varepsilon_{X_{0}}=\tilde{W}^{(\alpha)} \varepsilon_{X_{0}, C \Omega}^{\prime \prime} \quad \mu_{\tilde{\delta}} \text {-a.e., }
$$

which implies $\tilde{W}^{(\alpha)} \varepsilon_{X_{0}}=\tilde{W}^{(\alpha)} \varepsilon_{X_{0}, c \Omega}^{\prime \prime}$ on $R^{n} \times\left(-\infty, t_{0}-\delta\right)$ by Lemma 3.15 for $\tilde{L}^{(\alpha)}$. Therefore let $\delta \rightarrow 0$; then Proposition 2.10 yields

$$
\varepsilon_{X_{0}}=\varepsilon_{X_{0}, C \Omega}^{\prime \prime},
$$

which shows (4).

$(2) \rightarrow(1)$ : Let $\left(X_{m}\right)_{m=1}^{\infty}$ be an arbitrary sequence in $\Omega$ with $\lim _{m \rightarrow \infty} X_{m}$ $=X_{0}$. To show $\mathrm{w}^{*}-\lim _{m \rightarrow \infty} \varepsilon_{X_{m}, C \Omega}^{\prime \prime}=\varepsilon_{X_{0}}$, we may assume that $\left(\varepsilon_{X_{m}, C \Omega}^{\prime \prime}\right)_{m=1}^{\infty}$ converges vaguely. Put $\nu=\mathrm{w}^{*}-\lim _{m \rightarrow \infty} \varepsilon_{X_{m}, c \Omega}^{\prime \prime}$. For any $\mu \in M_{\alpha, c}$ whose support is compact, we have

$$
\begin{aligned}
\int \tilde{W}^{(\alpha)} \nu d \mu & =\lim _{m \rightarrow \infty} \int W^{(\alpha)} \mu d \varepsilon_{X_{m}, C \Omega}^{\prime \prime}=\lim _{m \rightarrow \infty} W^{(\alpha)} \mu_{C \Omega}^{\prime}\left(X_{m}\right) \\
& \geqq W^{(\alpha)} \mu_{C \Omega}^{\prime}\left(X_{0}\right)=W^{(\alpha)} \mu\left(X_{0}\right)=\int \tilde{W}^{(\alpha)} \varepsilon_{X_{0}} d \mu,
\end{aligned}
$$

so that $\tilde{W}^{(\alpha)} \nu \geqq \tilde{W}^{(\alpha)} \varepsilon_{X_{0}}$ a.e., that is, $\tilde{W}^{(\alpha)} \nu=\tilde{W}^{(\alpha)} \varepsilon_{X_{0}}$, which shows $\nu=\varepsilon_{X_{0}}$. Thus $X_{0}$ is regular. This completes the proof.

For any $(x, t) \in R^{n+1}$ and $k \in R$, we set

$$
\tau_{k}^{(\alpha)}(x, t)=\left(2^{k} x, 2^{2 \alpha k} t\right) .
$$

Remark 4.3. Let $u \in S_{\alpha}$ and $k \in R$ and put $v(X)=u\left(\tau_{k}^{(\alpha)} X\right)$. Then $v \in S_{\alpha}$.

We shall prove the following main theorem.

Theorem. Let $\Omega$ be an open set in $R^{n+1}$ and $X_{0} \in \partial \Omega$. If there exists a non-empty open set $\omega$ in $R^{n}$ such that $\alpha$-tusk $T_{X_{0}}^{(\alpha)}(\omega)$ of $\omega$ at $X_{0}$ is in 
$C \Omega$, then $X_{0}$ is regular for the Dirichlet problem of $L^{(\alpha)}$ on $\Omega$.

Proof. We may assume that $X_{0}$ is the origin 0 of $R^{n+1}$. By Proposition 4.1, we may assume that

$$
T_{0}^{(\alpha)}(\omega)=\left\{\left(p x,-p^{2 \alpha}\right) ; x \in \omega, 0<p<\infty\right\} .
$$

Put

$$
\begin{aligned}
& V=\{(x, t) ;-1<t<1,|x|<1\} \\
& V_{k}=\left\{\tau_{k}^{(\alpha)}(X) ; X \in V\right\} \\
& D=V \backslash \overline{T_{0}^{(\alpha)}(\omega)} \text { and } D_{k}=V_{k} \cap D(k: \text { integer })
\end{aligned}
$$

By Propositions 4.1 and 4.2, it suffices to show that 0 is regular on $D$. For any $\delta>0$, we can choose a positive integer $k$ such that

$$
\sup _{X \in V} \int_{C V_{k}} d \varepsilon_{X, C \bar{D}}^{\prime \prime}<\delta
$$

because for any $X \in \bar{D}, \varepsilon_{X}^{\prime \prime}, C \bar{D}-\varepsilon_{X}=\tilde{L}^{(\alpha)}\left(\tilde{W}^{(\alpha)} \varepsilon_{X, C \bar{D}}^{\prime \prime}-\tilde{W}^{(\alpha)} \varepsilon_{X}\right)$ in the sense of distribution, that is,

$$
\text { (4.2) } \varepsilon_{X, C \bar{D}}^{\prime \prime}=C_{n, \alpha}\left(\int_{R^{n}}\left(\tilde{W}^{(\alpha)} \varepsilon_{X}(y-z, t)-\tilde{W}^{(\alpha)} \varepsilon_{X, C \bar{D}}^{\prime \prime}(y-z, t)\right)|z|^{-n-2 \alpha} d z\right) d y d t
$$

in $C \bar{D}$. Put

$$
\begin{aligned}
u_{1}(X) & =\int_{C V} d \varepsilon_{X, C \bar{D}}^{\prime \prime}, \\
\beta & =\sup _{X \in V-1} \int_{C V} d \varepsilon_{X, C \bar{D}}^{\prime \prime},
\end{aligned}
$$

and

$$
u_{2}(X)=\beta \int_{C V_{-k-1}} d \varepsilon_{X, C \overline{D_{-k-1}}}^{\prime \prime}+(1-\beta) \int_{C V_{-1}} d \varepsilon_{X, C \overline{D_{-k-1}}}^{\prime \prime} .
$$

Then $\beta<1$. In fact, we take a sequence $\left(X_{m}\right)_{m=1}^{\infty} \subset V_{-1} \cap \bar{D}$ such that $\lim _{m \rightarrow \infty} \int_{C V} d \varepsilon_{X_{m}, C \bar{D}}^{\prime \prime}=\beta$. We may assume that $\left(\varepsilon_{X_{m}, C \bar{D}}^{\prime \prime}\right)_{m=1}^{\infty}$ converges vaguely to some $\nu \in \tilde{M}_{\alpha}$ as $m \rightarrow \infty$ and that $\left(X_{m}\right)_{m=1}^{\infty}$ converges to some point $X_{\infty}=$ $\left(x_{\infty}, t_{\infty}\right)$. Then

$$
\tilde{W}^{(\alpha)} \varepsilon_{X_{\infty}} \geqq \tilde{W}^{(\alpha)} \nu \quad \text { on } R^{n+1} .
$$

Since the family of the density of $\varepsilon_{X, c \bar{D}}^{\prime \prime}(X \in \bar{D})$ with respect to $d X$ is uniformly bounded on every compact set in $C \bar{D}$ (see (4.2) in this proof), we have 


$$
\tilde{W}^{(\alpha)} \varepsilon_{X_{\infty}}=\tilde{W}^{(\alpha)} \nu \text { on } C \bar{D} \text { and } \int d \nu=1
$$

Assume that $\beta=1 ; \int_{V} d \nu \leqq \liminf _{m \rightarrow \infty} \int_{V} d \varepsilon_{X_{m}}^{\prime \prime}, C \bar{D}=0$ and hence $\operatorname{supp}[\nu] \subset C V$. Since for any $\lambda \in M_{\alpha}, W^{(\alpha)} \lambda_{C \bar{D}}^{\prime}$ is continuous on $D$, the function $\int W^{(\alpha)} \lambda d \varepsilon_{X, C \bar{D}}^{\prime \prime}$ of $X$ is continuous on $D$ (see Proposition 3.8), so that the mapping $D \ni X \rightarrow \varepsilon_{X, C \bar{D}}^{\prime \prime}$ is vaguely continuous. Therefore Proposition 3.14 gives $X_{\infty} \in \overline{V_{-1}} \cap \partial D$, because if $X_{\infty} \in D$, then $\nu=\varepsilon_{X_{\infty}, C \bar{D}}^{\prime \prime}$, which contradicts

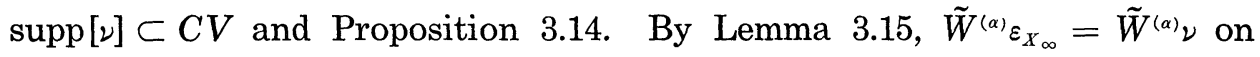
$\left\{(x, t) ; t<t_{\infty}\right\}$. Proposition 2.10 gives $\nu=\varepsilon_{X_{\infty}}$, which contradicts $\operatorname{supp}$ [ $\nu$ ] $\subset C V$. Thus $\beta<1$.

Let $\left(\phi_{m}\right)_{m=1}^{\infty}$ be an increasing sequence in $C_{K}^{\infty}\left(R^{n+1}\right)$ such that $0 \leqq \phi_{m} \leqq 1$, $\lim _{m \rightarrow \infty} \phi_{m}=1$ on $C V$ and that $\phi_{m}=0$ on $V_{-1}$. We write $\phi_{m}=W^{(\alpha)} \lambda_{m}$ with some signed measure $\lambda_{m}$. Then

$$
\int_{V-k-1}\left(\int_{C V} d \varepsilon_{Y, C \bar{D}}^{\prime \prime}\right) d \varepsilon_{X, c \overline{D-k-1}}^{\prime \prime}(Y) \leqq \lim _{m \rightarrow \infty} \int_{V_{-k-1}} W^{(\alpha)} \lambda_{m, C \bar{D}}^{\prime} d \varepsilon_{X, c \overline{D-k-1}}^{\prime \prime},
$$

where $\lambda_{m, C \bar{D}}^{\prime}=\left(\lambda_{m}^{+}\right)_{C \bar{D}}^{\prime}-\left(\lambda_{m}^{-}\right)_{C \bar{D}}^{\prime}$. Since Corollary 3.13 gives

$$
\left(\varepsilon_{X, C \overline{D-k-1}}^{\prime \prime}\right)_{C \bar{D}}^{\prime \prime}=\left.\varepsilon_{X, C \overline{D_{-k-1}}}^{\prime \prime}\right|_{V-k-1}+\left(\left.\varepsilon_{X, C \overline{D_{-k-1}}}^{\prime \prime}\right|_{C V-k-1}\right)_{C \bar{D}}^{\prime \prime}
$$

we have

$$
\int_{V_{-k-1}} W^{(\alpha)} \lambda_{m, C \bar{D}}^{\prime} d \varepsilon_{X, c \overline{D_{-k-1}}}^{\prime \prime}=\int_{V_{-k-1}} W^{(\alpha)} \lambda_{m} d\left(\varepsilon_{X, c \overline{D_{-k-1}}}^{\prime \prime}\right)=0 .
$$

Let $\left(\phi_{m}\right)_{m=1}^{\infty}$ be a sequence in $C_{K}^{\infty}\left(R^{n+1}\right)$ such that $0 \leqq \phi_{m} \leqq 1$ and $\lim _{m \rightarrow \infty} \phi_{m}(X)$ $=1$ on $C V$ and $=0$ on $V$. Since $\phi_{m}=W^{(\alpha)} \lambda_{m}$ with some signed measure $\lambda_{m}$, by Proposition 3.8, we have, for $X \in V_{-k-1}$,

$$
\begin{aligned}
u_{1}(X) & =\lim _{m \rightarrow \infty} \int \phi_{m} d \varepsilon_{X, C \bar{D}}^{\prime \prime}=\lim _{m \rightarrow \infty} \iint \phi_{m} d \varepsilon_{Y, C \bar{D}}^{\prime \prime} d \varepsilon_{X, C \overline{D-k-1}}^{\prime \prime}(Y) \\
& =\int\left(\int_{C V} d \varepsilon_{Y, C \bar{D}}^{\prime \prime}\right) d \varepsilon_{X, C \overline{D-k-1}}^{\prime \prime}(Y) \\
& =\int_{C V-k-1}\left(\int_{C V} d \varepsilon_{Y, C \bar{D}}^{\prime \prime}\right) d \varepsilon_{X, C \overline{D-k-1}}^{\prime \prime}(Y) \\
& \leqq u_{2}(X) \leqq \beta u_{1}\left(\tau_{k+1}^{(\alpha)}(X)\right)+(1-\beta) \delta
\end{aligned}
$$

Thus we obtain inductively

$$
\limsup _{X \rightarrow 0} u_{1}(X) \leqq \sum_{k=0}^{\infty} \beta^{k}(1-\beta) \delta=\delta,
$$

which gives 


$$
\lim _{X \rightarrow 0} u_{1}(X)=0
$$

By Proposition 3.14, we can choose $f \in C_{K}\left(R^{n+1}\right)$ such that $0 \leqq f \leqq 1$, $\operatorname{supp}[f] \subset C V$ and that

$$
u(X)=\int f(Y) d \varepsilon_{X, C \bar{D}}^{\prime \prime}(Y)>0 \quad \text { on } D .
$$

Take $\phi \in C_{K}\left(R^{n+1}\right)$ such that $\operatorname{supp}[\phi] \subset D, \phi \geqq 0, \operatorname{Int}(\operatorname{supp}[\phi]) \cap\{(x, t) ; t<0\}$ $\neq \phi$ and that $W^{(\alpha)}(\phi d X) \leqq u$ on $\operatorname{supp}[\phi]$. For any open set $\omega \supset C D$, we put $\omega_{0}=\{X ; \phi(X)>0\} \cup \omega$. Let $\left(\omega_{m}\right)_{m=1}^{\infty}$ be an exhaustion of $\omega_{0}$. Then we have

$$
\begin{aligned}
& W^{(\alpha)}(\phi d X)(X)-W^{(\alpha)}(\phi d X)_{\omega}^{\prime}(X) \\
& \quad=\int\left(W^{(\alpha)}(\phi d X)-W^{(\alpha)}(\phi d X)_{\omega}^{\prime}\right) d \varepsilon_{X, \omega_{0}}^{\prime \prime} \\
& \quad=\lim _{m \rightarrow \infty} \int\left(W^{(\alpha)}(\phi d X)-W^{(\alpha)}(\phi d X)_{\omega}^{\prime}\right) d \varepsilon_{X, \omega_{m}}^{\prime \prime} \\
& \quad \leqq \lim _{m \rightarrow \infty} \int u d \varepsilon_{X, \omega_{m}}^{\prime \prime}=\lim _{m \rightarrow \infty} \int\left(\int f{\varepsilon_{Y}^{\prime \prime}, c \bar{D}}^{\prime \prime}\right) d \varepsilon_{X, \omega_{m}}^{\prime \prime}(Y)=u(X) .
\end{aligned}
$$

By Lemma 3.5, we have

$$
W^{(\alpha)}(\phi d X)(X)-W^{(\alpha)}(\phi d X)_{C D}^{\prime}(X) \leqq u(X) \quad \text { on } R^{n+1},
$$

which implies

$$
\lim _{X \in \Omega, X \rightarrow 0} W^{(\alpha)}(\phi d X)_{C D}^{\prime}(X)=W^{(\alpha)}(\phi d X)(0)
$$

Hence

$$
W^{(\alpha)}(\phi d X)_{C D}^{\prime}(0)=W^{(\alpha)}(\phi d X)(0)
$$

(see, for example, the proof of $(1) \rightarrow(3)$ in Proposition 4.2). By Proposition 4.2 , (3), 0 is regular for the Dirichlet problem of $L^{(\alpha)}$ on $D$. This completes the proof.

Acknowledgement. The authors are thankful to the referee for his suggestions.

\section{REFERENCES}

[1] C. Berg and G. Forst, Potential theory on locally compact abelian groups, SpringerVerlag, Berlin Heiderberg New York, 1975.

[2] G. Choquet and J. Deny, Sur l'equation de convolution $\mu=\mu * \sigma$, C. R. Acad. Sci., 250 (1960), 799-801. 
[3] E. Effros and J. Kazdan, On the Dirichlet problem for the heat equation, Indiana U. Math. J., 20 (1971), 683-693.

[4] J. Elliot, Dirichlet spaces and integro-differential operators, Part I, Illinois J. Math., 9 (1965), 87-98.

[5] L. Evans and R. Griepy, Wiener's criterion for the heat equation, Arch. Rational Mech. Anal., 78 (1982), 293-314.

[6] L. L. Helms, Introduction to potential theory, Wiley-Interscience, New York, 1969.

[ 7 ] C. R. Herz, Analyse harmonique à plusiers variables, Sém. Math. d'Orsay, 1965/66.

M. Itô

Department of Mathematics

College of General Education

Nagoya University

Chikusa-ku, Nagoya, 464,

Japan

M. Nishio

Department of Mathematics

School of Sciences

Nagoya University

Chikusa-ku, Nagoya, 464,

Japan 